\title{
COUNTEREXAMPLES TO SEVERAL PROBLEMS ON THE FACTORIZATION OF BOUNDED LINEAR OPERATORS
}

\author{
N. GHOUSSOUB AND W. B. JOHNSON ${ }^{1}$
}

\begin{abstract}
For every $1 \leq p<\infty$, there exist a Banach lattice $X_{p}$ and a lattice homomorphism $T_{p}$ from $X_{p}$ onto $c_{0}$ which satisfy:

(1) $T_{p}$ does not preserve an isomorphic copy of $c_{0}$.

(2) $T_{p}$ is a Radon-Nikodym operator.

(3) $T_{1}$ maps weakly Cauchy sequences into norm convergent sequences.

(4) If $T_{p}$ is written as the product of two operators, then one of them preserves a copy of $c_{0}$.
\end{abstract}

0. Introduction. In [4], T. Figiel and the authors constructed the spaces $X_{p}$ and the lattice homomorphisms $T_{p}$ from $X_{p}$ onto $c_{0}$ mentioned in the Abstract and verified (1). It turns out that these examples also can be used to answer several questions on the factorization of operators between Banach spaces of the following kind (see Pietsch's book [13] for a complete discussion of this type of problem): Given a property $(\mathrm{P})$ for operators which determines a Banach ideal of operators, is it true that every operator with property $(\mathrm{P})$ can be factored as the product of two operators with property $(\mathrm{P})$ ? In this note we show that the answer is negative for the properties $(\mathrm{P})=$ Radon-Nikodym and $(P)=$ does not preserve a copy of $c_{0}$, thus answering, respectively, questions of Stegall $[\mathbf{1 5}]$ and Pełczynski (see also Pietsch [13, Conjectures 2.6.2 and 3.1.9]).

Unexplained notation can be found in the books of Lindenstrauss and Tzafriri [ 7 , 8]. Here we note only that an operator $T$ from a Banach space $X$ is called a RadonNikodym operator if $T$ takes $X$-valued bounded martingales into martingales which converge pointwise almost everywhere. An operator $T$ from $X$ preserves a copy of $c_{0}$ if there is a subspace of $X$ isomorphic to $c_{0}$ such that the restriction of $T$ to the subspace is an isomorphism.

We thank Michel Talagrand for several useful conversations. In particular, the result (2) that $T_{p}$ is a Radon-Nikodym operator is due to Talagrand with essentially the proof that we present here.

I. The counterexamples. We now recall the space constructed in [4]. Let $c$ be the space of converging sequences and set $X=l_{1}(c)$; that is, the space of doubly-indexed sequences $a=\left(a_{i, j}\right)$, where $i=1,2, \ldots ; j=1,2, \ldots, \omega$ such that

and

$$
\lim _{j \rightarrow \infty} a_{i, j}=a_{i, \omega} \text { for } i=1,2, \ldots
$$

$$
\|a\|_{X}=\sum_{i=1}^{\infty} \sup _{j}\left|a_{i, j}\right|<\infty .
$$

Received by the editors August 1, 1983.

1980 Mathematics Subject Classification. Primary 46M35, 47A68.

Key words and phrases. Factorization of linear operators.

${ }^{1}$ Supported in part by NSF MCS-7903042. 
Define $f_{n}$ in $X$ by

$$
\left(f_{n}\right)_{i, j}= \begin{cases}1 & \text { if } i \leq n \leq j \leq \omega \\ 0 & \text { otherwise }\end{cases}
$$

Let $X_{0}$ be the dense sublattice of $X$ consisting of those vectors $a=\left(a_{i, j}\right)$ whose rows are eventually zero; i.e., for some $n, a_{i, j}=0$ for all $i \geq n$ and all $j=1,2, \ldots$.

On $X_{0}$ we define a new lattice norm by setting

$$
\|x\|_{1}=\inf \left\{\|g\|_{X}+\sum_{i=1}^{n}\left|\alpha_{i}\right|:|x| \leq g+\sum_{i=1}^{n} \alpha_{i} f_{i}\right\} .
$$

Let $\left(X_{1},\|\|_{1}\right)$ be the completion of $\left(X_{0},\|\|_{1}\right)$ and for $1<p<\infty$, let $\left(X_{p},\|\|_{p}\right)$ be the completion of the $p$-convexification of $\left(X_{0},\|\|_{1}\right)$; that is, for $x \in X_{0}$

$$
\|x\|_{p}=\left\||x|^{p}\right\|_{1}^{1 / p}
$$

Define an operator $T: X_{0} \rightarrow c_{0}$ by setting $T a=\left(a_{i, \omega}\right)_{i=1}^{\infty}$. In [4] it was shown that for each $1 \leq p<\infty, T$ extends to a norm one operator from the space $X_{p}$ into $c_{0}$ (call the extension $T_{p}$ ).

The following properties were proved in [4].

(i) $X_{p}$ contains no subspace isomorphic to $l_{1}$ for $p>1$.

(ii) The operator $T_{p}$ is a lattice homomorphism and a quotient map from $X_{p}$ onto $c_{0}$, and $T_{p}$ is strictly singular; in particular, $T_{p}$ satisfies condition (1) in the Abstract.

We now verify condition (4) in the Abstract: First, observe that if $x$ is any vector in $X$ which is supported on a single row, then for each $1 \leq p<\infty,\|x\|_{p}=\|x\|_{X}$; i.e., the vectors in $X_{p}$ supported on any given row form a Banach lattice which is isometrically isomorphic to $c$. Now suppose that $R: X_{p} \rightarrow Y$ and $S: Y \rightarrow c_{0}$ are bounded operators, $T_{p}=S R$, and $R$ does not preserve a copy of $c_{0}$. It is well known (combine the remark after Lemma 1.a.5 with Propositions 2.a.1 and 1.a.12 in [7]) that an operator from $c$ either preserves a copy of $c_{0}$ or is compact, so the above observation yields that the restriction of $R$ to the vectors supported on the first $n$ rows is a compact operator. Consequently, letting $x(n, i)$ be the indicator function of the set $\{(n, j): i \leq j \leq \omega\}$, we get that $R x(n, j)$ converges to a limit, say, $y_{n}$, in $Y$ as $j \rightarrow \infty$. Now $S y_{n}=T x(n, i)$ is the $n$th unit vector in $c_{0}$, so we have that for any scalars $\left(\alpha_{n}\right)$,

$$
\left\|\sum \alpha_{n} y_{n}\right\| \geq\|S\|^{-1} \max \left|\alpha_{n}\right| \text {. }
$$

Moreover, for any $i \geq n$ and any scalars $\left(\alpha_{k}\right)_{k=1}^{n}$, we see that

$$
\left|\sum_{k=1}^{n} \alpha_{k} x(k, i)\right| \leq \max \left\{\left|\alpha_{k}\right|: 1 \leq k \leq n\right\} f_{n},
$$

and hence

$$
\left\|\sum \alpha_{n} y_{n}\right\| \leq\|R\| \max \left|\alpha_{n}\right|\left\|f_{n}\right\|_{p}=\|R\| \max \left|\alpha_{n}\right| \text {. }
$$

That is, the sequence $\left(y_{n}\right)$ is equivalent to the unit vector basis of $c_{0}$ and the restriction of $T_{p}$ to its closed linear span is an isomorphism. This completes the proof of (4).

In order to check the other properties of $T_{p}$, we need a technical proposition about $X_{p}$. 
PROPOSITION 1. Suppose that $0=k_{0}<n_{1} \leq k_{1}<n_{2} \leq k_{2}<n_{3} \leq \cdots, A_{r}=$ $\left\{(i, j): k_{r-1}<i \leq n_{r} \leq j \leq k_{r}\right\}$ and $d(r)=\operatorname{card} A_{r}$. Let $E_{r}$ be the vectors in $X$ which are supported on $A_{r}$. Then $\left[\left(E_{r}\right)_{r=1}^{\infty}\right]$ is, in $X_{p}$, isometrically lattice isomorphic to $\left(\sum_{r} l_{\infty}^{d(r)}\right)_{p}$.

ProOF. First note that if $x$ is a vector in $X$ which is supported on a strip of the form

$$
S_{n}=\{(i, j): 1 \leq i \leq n \leq j \leq \omega\}
$$

then for all $1 \leq p<\infty,\|x\|_{p}=\|x\|_{\infty}$. Indeed, it is easy to see (and was pointed out in [4]) that the evaluation functionals, defined for $1 \leq i<\infty, 1 \leq j \leq \omega$ by $e(i, j)(x)=x_{i, j}$, are norm one functionals on each space $X_{p}$, hence $\|x\|_{\infty} \leq\|x\|_{p}$ for every $x$ in $X$. But if $x$ is supported on the strip $S_{n}$ then $|x| \leq\|x\|_{\infty} f_{n}$ and $\left\|f_{n}\right\|_{p}=1$, so $\|x\|_{p} \leq\|x\|_{\infty}$.

Thus for each $r, E_{r}$ is lattice isometric to $l_{\infty}^{d(r)}$. Since $X_{p}$ is $p$-convex, to complete the proof it is enough to check that if $x$ is supported on $\bigcup_{r=1}^{n} E_{r}$, then

$$
\|x\|_{p}^{p} \geq \sum_{r=1}^{n}\left|1_{E_{r}} x\right|^{p} .
$$

Since the $E_{r}$ 's are disjoint, it is enough to prove (*) only for $p=1$. This case is an immediate consequence of the following

ClaIM. If $\left(i_{r}, j_{r}\right)$ is in $E_{r}$ for $1 \leq r<\infty$, then $e\left(i_{r}, j_{r}\right)$ is, in $X_{1}^{*}$, isometrically equivalent to the unit vector basis for $c_{0}$.

To prove the Claim, note that

$$
\left\|\sum a_{r} e\left(i_{r}, j_{r}\right)\right\|_{X_{i}^{*}} \geq \max \left|a_{r}\right|
$$

because the $e\left(i_{r}, j_{r}\right)$ 's are disjoint vectors in a Banach lattice. On the other hand, $i_{r} \neq i_{s}$ if $r \neq s$ so that

$$
\left\|\sum a_{r} e\left(i_{r}, j_{r}\right)\right\|_{X^{*}}=\max \left|a_{r}\right|
$$

Moreover, for each $n,\left(i_{r}, j_{r}\right)$ is in at most one strip $S_{n}=\operatorname{supp} f_{n}$, hence, for each $n=1,2,3, \ldots$,

$$
\sum\left|a_{r}\right| e\left(i_{r}, j_{r}\right)\left(f_{n}\right) \leq \max \left|a_{r}\right|
$$

This shows that

$$
\left\|\sum a_{r} e\left(i_{r}, j_{r}\right)\right\|_{X_{\mathrm{i}}^{*}} \leq \max \left|a_{r}\right|
$$

and completes the proof of the Claim and Proposition 1.

Condition (3) in the Abstract is an immediate consequence of part (a) of Proposition 2. Moreover, Proposition 2 provides a proof of condition (1) in the Abstract which is probably simpler than that given in $[\mathbf{4}]$.

Proposition 2. Suppose that $\left(x_{n}\right)$ is a bounded sequence in $X_{p}$ and $\left(T_{p} x_{n}\right)$ is not relatively compact in $c_{0}$.

(a) If $p=1,\left(x_{n}\right)$ has a subsequence which is equivalent to the unit vector basis for $l_{1}$ and which spans a complemented subspace of $X_{1}$. 
(b) If $1<p<\infty,\left(x_{n}\right)$ has a subsequence $\left(y_{n}\right)$ such that $\left(y_{2 n}-y_{2 n-1}\right)$ is equivalent to the unit vector basis for $l_{p}$ and spans a complemented subspace of $X_{p}$.

PROOF. There is no loss of generality in assuming that each $x_{n}$ is in $X_{0}$. Since $\left(T_{p} x_{n}\right)$ is a bounded sequence in $c_{0}$ which is not relatively compact, we can assume by passing to a subsequence of the $x_{n}$ 's that

$$
\left|\left(x_{n}\right)_{i(n), \omega}\right|>\mu>0 \quad \text { with } i(n) \rightarrow \infty
$$

By passing to a further subsequence, we can pick $j(n)$ so that

$$
\left|\left(x_{n}\right)_{i(n), j(n)}\right|>\mu \quad \text { and } \quad i(n) \leq j(n)<i(n+1)
$$

Thus, by Proposition 1 , the evaluation functionals $e(i(n), j(n))$ are, in $X_{p}^{*}$, isometrically equivalent to the unit vector basis for $l_{q}(1 / p+1 / q=1)$, and hence the operator $L: X_{p} \rightarrow l_{p}$ defined by

$$
(L x)(n)=e(i(n), j(n))(x)
$$

is a norm one operator from $X_{p}$ into $l_{p}$ so that the $n$th coordinate of $L x_{n}$ is larger than $\mu$. Therefore, in the case $p=1$, by (an easy special case of) the results in $[\mathbf{1 4}],\left(L x_{n}\right)$ has a subsequence which is equivalent to the unit vector basis of $l_{1}$ and which spans a complemented subspace of $l_{1}$. From this it is easy to check that the corresponding subsequence of $\left(x_{n}\right)$ satisfies the conclusions of $(\mathrm{a})$.

Since we do not use (b) in the sequel, we only sketch the idea for completing the proof in the case $p>1$. First we pass to successive differences of a subsequence of the $x_{n}$ 's (call it $\left(z_{n}\right)$ ) so that $\left(z_{n}\right)$ converges weakly to 0 in $X_{p}$ and $\left(L z_{n}\right)$ is equivalent to the unit vector basis for $l_{p}$ and spans a complemented subspace of $L_{p}$. This gives a lower $l_{p}$-estimate for linear combinations of the $z_{n}$ 's. Now we use the special form of $X_{p}$ to justify that a subsequence of the $z_{n}$ 's is a small perturbation of a sequence of the form $\left(u_{n}+v_{n}\right)$, where the $u_{n}$ 's are supported on $\{(i, j): j<\omega\}$ and where each of the bounded subsequences $\left(u_{n}\right)$ and $\left(v_{n}\right)$ consists of disjointly supported vectors. The upper $l_{p}$ estimate for the corresponding subsequence of $\left(z_{n}\right)$ then follows from the $p$-convexity of $X_{p}$.

We turn now to the verification of condition (2) in the Abstract. (This result is due to M. Talagrand.)

Given a bounded martingale $f^{n}:[0,1] \rightarrow X_{p}$, we need to show that $\left(T_{p} f^{n}\right)$ is almost surely norm convergent. It is well known that it is sufficient to check this only for the case that each $f^{n}$ is a simple function, so we assume this extra condition.

Let $Y$ be the weak*-limit in $l_{\infty}$ of $\left(T_{p} f^{n}\right)$. It is also well known that if $\left(T_{p} f^{n}\right)$ is not almost surely norm convergent in $c_{0}$, then it is also not almost surely weakly convergent, so there is a subset $C$ of $[0,1]$ with positive measure and a $\mu>0$ so that for each $t$ in $C, d\left(Y(t), c_{0}\right)>\mu$. By replacing $C$ with a smaller subset of positive measure, we can assume that there is an increasing sequence $\left(m_{k}\right)$ so that for each $t$ in $C$, 
(i) $\sup \left\{|Y(t)(m)|: m_{k}<m \leq m_{k+1}\right\}>\mu$.

Since for each coordinate $m$, the real valued martingale $\left(T_{p} f^{n}(m)\right)$ converges to $Y(m)$, we can assume, by replacing $C$ with a smaller set of positive measure, that there is an increasing sequence $\left(n_{k}\right)$ so that for each $t$ in $C$ and $k=1,2,3, \ldots$,

(ii) $\sup \left\{\left|T_{p} f^{n_{k}}(t)(m)-Y(t)(m)\right|: 1 \leq m \leq m_{k}\right\}<\mu / 4$.

Since each $f^{n}$ is a simple function, for each $k=1,2,3, \ldots$, we can take $j_{k}>m_{k+1}$ so that for all $t$ in $C$,

(iii) $\sup \left\{\left|f^{n_{k}}(t)\left(m, j_{k}\right)-T_{p} f^{n_{k}}(t)(m)\right|: 1 \leq m \leq m_{k+1}\right\}<\mu / 4$.

Combining (i), (ii) and (iii) we get for all $t$ in $C$ that

$$
\sup \left\{\left|f^{n_{k}}(t)\left(m, j_{k}\right)\right|: m_{k}<m \leq m_{k+1}\right\}<\mu / 4 .
$$

Let $(k(r))$ be a sequence so that $m_{k(r+1)}>j_{k(r)}$ and set

$$
A_{r}=\left\{\left(i, j_{k(r)}\right): m_{k(r)}<i \leq m_{k(r)+1}\right\} .
$$

Let $P_{r}$ (respectively $P$ ) be the band projection from $X_{p}$ onto the functions supported on $A_{r}$ (respectively on the union of all the $A_{r}$ 's). By Proposition 1, the range of $P$ is an $l_{p}$-sum of finite-dimensional spaces and thus has the Radon-Nikodym property. Therefore, the martingale $\left(P f^{n}\right)$ is norm convergent almost surely to, say, $f$, and thus for almost all $t,\left\|P_{r} f(t)\right\|<\mu / 4$ for large $r$, while for $t$ in $C$ and all $r,\left\|P_{r} f^{n_{k(r)}}(t)\right\|>\mu / 4$, which is a contradiction.

REMARK. It was noted in [4] that for $p>1, T_{p}$ is an example of a nonweakly compact operator from a Banach lattice not containing a complemented copy of $l_{1}$ which does not preserve a copy of $c_{0}$. This showed that Pełczynski's theorem $[\mathbf{1 1}]$ (unlike some other results) that nonweakly compact operators from $C(K)$ spaces preserve a copy of $c_{0}$ does not generalize to operators from such lattices. On the other hand, it is shown in [5] that Grothendieck's [6] forerunner to Pełczynski's theorem does have a lattice analogue; in fact, it is proved that every operator from a Banach lattice not containing a complemented copy of $l_{1}$ into a Banach space not containing a copy of $c_{0}$ is weakly compact.

\section{REFERENCES}

1. W. J. Davis, T. Figiel, W. B. Johnson and A. Pełczynski, Factoring weakly compact operators, J. Funct. Anal. 17 (1974), 311-327.

2. J. Diestel and J. J. Uhl, Vector measures, Math. Surveys, no. 15, Amer. Math. Soc., Providence, R.I., 1977

3. T. Figiel, W. B. Johnson and L. Tzafriri, On Banach lattices and spaces having local unconditional structure with applications to Lorentz function spaces, J. Approx. Theory 13 (1975), 395-412.

4. T. Figiel, N. Ghoussoub and W. B. Johnson, On the structure of non weakly compact operators on Banach lattices, Math. Ann. 257 (1981), 317-334.

5. N. Ghoussoub and W. B. Johnson, On subspaces of Banach lattices not containing $C(\Delta)$ (in preparation).

6. A. Grothendieck, Sur les applications linéaires faiblement compactes d'espaces du type $C(K)$, Canad. J. Math. 5 (1953), 129-173.

7. J. Lindenstrauss and L. Tzafriri, Classical Banach spaces. I. Sequence spaces, Ergeb. Math. Grenzgeb., vol. 92, Springer, Berlin, 1977.

8. _ C Classical Banach spaces. II. Function spaces, Ergeb. Math. Grenzgeb., vol. 97, Springer, Berlin, 1979.

9. H. P. Lotz and H. P. Rosenthal, Embeddings of $C(\Delta)$ and $L^{1}[0,1]$ in Banach lattices, Israel J. Math. 31 (1978), 169-179. 
10. P. Meyer-Nieberg, Zur schwachen Kompaktheir in Banachverbanden, Math. Z. 134 (1973), 303316.

11. A. Pełczynski, Projections in certain Banach spaces, Studia Math. 19 (1960), 209-228.

12. __ Banach spaces on which every unconditionally converging operator is weakly compact, Bull. Acad. Polon. Sci. Sér. Sci. Math. Astronom. Phys. 10 (1962).

13. A. Pietsch, Operator ideals, North-Holland, Amsterdam, 1980.

14. H. P. Rosenthal, On relatively disjoint families of measures, with some applications to Banach space theory, Studia Math. 37 (1971), 13-36.

15. C. Stegall, The Radon-Nikodym property in conjugate Banach spaces. II, Trans. Amer. Math. Soc. 264 (1981), 507-519.

Department of Mathematics, Ohio State University, Columbus, Ohio 43210

Department of Mathematics, University of British Columbia, Vancouver, B.C., CANADA 